\title{
A AQUISIÇÃO FONOLÓGICA DO INGLÊS COMO L2 À LUZ DA OT: OS VERBOS CONTENDO A MARCA «-ED» - Ubiratã Kickhöfel Alves -
}

RESUMO: This paper focuses on the acquisition, by Brazilian Portuguese speakers, of both the syllabic structure and the sonority of the coda segments of the English verbs containing the morpheme "-ed". The analysis which was developed under the framework of Optimality Theory was able to show the evolution of the learners' constraint ranking from the initial state $H_{0}$ to the hierarchy which led to native-like production. The analysis has also shown that, regarding the acquisition of the syllabic structure, the production of native-like forms implied not only the demotion of constraints, but also the abandonment of an input originated from the written form, and the subsequent acquisition of a new underlying representation which was able to lead to the production of the target structures. In this regard, the analysis has revealed that, as for L2 acquisition, variable output forms do not need to be solely originated from the constraint ranking, given the fact that such outputs may also come from the existence of more than one input representation maintained by the same learner.

PALAVRAS-CHAVE: Aquisição do inglês como L2. Codas Complexas. Morfema "-ed". Teoria da Otimidade.

\section{INTRODUÇÃO}

No que diz respeito ao processo de aquisição de uma segunda língua, a Teoria da Otimidade (OT - Prince \& Smolensky 1993) revela-se como um modelo de análise poderoso em casos de acompanhamento longitudinal dos diversos estágios de interlíngua por que passam os aprendizes. Até se mostrar capaz de chegar à forma alvo, o aprendiz passará por uma série de hierarquias intermediárias entre a gramática da sua L1 e o ranqueamento capaz de levar à produção das formas semelhantes à da L2. Todos esses estágios, tendo-se por base de análise o modelo da OT, são decorrentes de hierarquias distintas. Tendo-se uma hierarquia de origem (L1) e uma de destino (aquela capaz de levar a outputs semelhantes às formas-alvo), pode-se assim determinar, na categorizarão das diversas hierarquias que caracterizam estágios intermediários entre esses dois estados hierárquicos, o quão perto ou longe cada um desses sistemas intermediários se encontra de tal hierarquia-alvo, permitindo-se, dessa forma, apontar quais formas de saída indicam um estágio mais ou menos avançado de aquisição de segunda língua. As afirmações acima feitas originam-se em função do que diz o próprio algoritmo de aprendizagem de Tesar \& Smolensky (1996, 2000) para a aquisição de primeira língua, a partir do qual se depreende que adquirir uma segunda língua implica demover restrições a partir da hierarquia da L1, recursivamente, até se atingir um ranqueamento capaz de levar a outputs ótimos semelhantes àqueles encontrados na língua-alvo.

O presente trabalho visa a evidenciar que, no processo de aquisição da L2, nem sempre a demoção de restrições representa uma condição por si só suficiente para levar os aprendizes à produção de formas-alvo. Para isso, propõe-se realizar um estudo da aquisição fonológica dos verbos do inglês que exibem a marca de passado/particípio passado regular, caracterizados pela presença de um segmento plosivo coronal, cuja sonoridade depende da consoante final da raiz do verbo (como em lived [livd] e missed [mist]). A produção semelhante ao falar nativo desses verbos implica, para o aprendiz brasileiro, duas dificuldades: a primeira diz respeito à aquisição de padrões silábicos não permitidos pela

\footnotetext{
${ }^{1}$ Ubiratã Kickhöfel Alves é doutorando em Lingüística Aplicada da Pontifícia Universidade Católica do Rio Grande do Sul. O presente artigo representa uma síntese da dissertação de mestrado intitulada O papel da instrução explícita na aquisição fonológica do inglês como LE: evidências fornecidas pela Teoria da Otimidade, defendida em março de 2004 na Universidade Católica de Pelotas (UCPel), sob orientação da Profa. Dr. Carmen Lúcia Matzenauer.
} 
sua L1, e a segunda concerne à sonoridade do segmento plosivo coronal final.

As duas fontes de dificuldade acima descritas servirão para o argumento que será comprovado através da análise via OT: enquanto que, tanto no que diz respeito à aquisição dos padrões silábicos da L2 quanto à capacidade de produção do segmento plosivo final com o traço [+son], é necessário que o aprendiz demova restrições, no caso da produção das codas complexas, é ainda preciso, além do processo de demoção acima apontado, a aquisição de uma nova forma subjacente, sem a qual o aprendiz não será capaz de produzir as seqüências consonantais finais que representam o foco do presente estudo. Afirmase, assim, que a representação de input mantida pelos aprendizes, em um estágio inicial de aquisição, já se mostrava capaz de levar à produção das formas-alvo no que diz respeito à questão da sonoridade da plosiva coronal final. Tal representação, entretanto, mostravase de caráter inadequado para a produção da estrutura silábica semelhante ao falar nativo. A análise a ser realizada à luz da OT, neste trabalho, visa a demonstrar argumentos que justifiquem a afirmação aqui feita.

\section{FENÔMENO ANALISADO}

Conforme afirmado, o estudo visa a verificar a produção da marca de passado simples/particípio passado dos verbos regulares do inglês, caracterizados pelo acréscimo do fonema /d/ à raiz do verbo, fonema esse que, em sua manifestação de superfície, pode ser produzido sob sua forma surda [t] ou sonora [d], dependentemente do valor positivo ou negativo do vozeamento da consoante final da raiz.

São apresentadas, em (1), as estruturas de coda silábica do inglês compostas por duas consoantes, cuja produção é analisada por este trabalho.

(1)

\begin{tabular}{|c|c|c|c|}
\hline $\begin{array}{l}\text { [pt] stopped } \\
\text { [kt] picked } \\
\text { [ft] laughed } \\
{\left[\int \mathrm{t}\right] \text { finished }} \\
\left.\text { [t } \int \mathrm{t}\right] \text { watched } \\
\text { [bd]robbed } \\
\text { [gd] unplugged } \\
\text { [dzd] judged } \\
\text { [vd] believed }\end{array}$ & 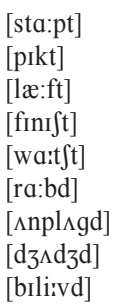 & $\begin{array}{l}\text { [nd] banned } \\
\text { [md] bloomed } \\
\text { [st] missed } \\
\text { [ld] called } \\
\text { [rd] remembered } \\
\text { [jd] played } \\
\text { [wd] narrowed }\end{array}$ & $\begin{array}{l}\text { [bæ:nd] } \\
\text { [blu:md] } \\
\text { [mist] } \\
\text { [ka:ld] } \\
\text { [rimembərd] } \\
\text { [plejd] } \\
\text { [nærowd] }\end{array}$ \\
\hline
\end{tabular}

Ao se considerar a produção das estruturas silábicas aqui descritas por parte de falantes nativos do português brasileiro, deve-se lembrar que a condição de coda do português admite qualquer soante, como /N/, /1/, /r/, e, além disso, a fricativa alveolar, o único elemento não soante permitido em coda (cf. Bisol 1999). Quanto à formação de codas complexas, no português a estrutura de coda pode apresentar duas consoantes, desde que a primeira seja uma soante e a segunda seja necessariamente um segmento fricativo alveolar. Ressalta-se, assim, a necessidade de aquisição dos padrões de coda complexa da L2.

No que diz respeito à questão da sonoridade da plosiva coronal final que caracteriza a marca de passado simples do inglês, tal questão pode ser entendida a partir dos exemplos em (2).

(2) (a)

[ stapt ] stopped

[ læft ] laughed (b)

[ rabd ] robbed [ livd ] lived
Nota-se a existência de um processo de assimilação progressiva do traço [ \pm sonoro], por meio do qual o morfema de passado copia tal traço do segmento final da raiz do verbo. Considera-se que a forma subjacente do morfema "-ed" em inglês caracteriza-se pela plosiva coronal exibindo o traço [+son], de modo que tal plosiva seja dessonorizada quando antecedida por um segmento surdo, como pode ser visto a partir dos exemplos em (02a). A dificuldade, por parte do falante nativo de português, é justamente produzir as plosivas finais com a presença da propriedade sonoridade, uma vez que há uma tendência de produzir tais segmentos com o traço [-son].

\section{METODOLOGIA}

O estudo contou com sete informantes, todos acadêmicos do curso de Letras - Habilitação em Língua Inglesa - de uma universidade do interior do Rio Grande do Sul. Todos os alunos cursavam uma disciplina em que recebiam instrução formal acerca dos principais fenômenos fonético/ fonológicos do português e do inglês.

$\mathrm{O}$ estudo contou com três momentos de coleta de dados, um anterior e dois posteriores a um trabalho, desenvolvido na disciplina em questão, de instrução explícita acerca da produção fonética da marca "-ed". Os três momentos de coleta, dessa forma, tinham o objetivo de determinar a 
necessidade de tal trabalho de instrução, bem como os seus efeitos benéficos e o seu possível caráter duradouro. Dado o objetivo do estudo de verificar a produção do item-alvo em contextos diversos, que propiciassem diferentes graus de monitoração do aprendiz, foram propostos instrumentos de coleta de dados que contavam tanto com tarefas de leitura de textos e frases, como também com oportunidades de produção oral em situações de uso espontâneo da fala.

\section{AS FORMAS DE OUTPUT PRODUZIDAS}

Para facilitar a análise que será a partir de então desenvolvida, o presente trabalho propõe a categorização dos verbos em dois grupos em função da estrutura silábica, conforme apresentado em (3).

(3)

Grupo A - Verbos caracterizados por codas complexas cujo penúltimo segmento não é permitido, no $\mathrm{PB}$, em posição de coda. Exemplos: lived [livd], watched [watst], stopped [sto:pt], laughed [læ:ft].

Grupo B - Verbos caracterizados por codas complexas cujo penúltimo segmento é permitido, em posição de coda, pela L1 (/l, r, N, S/). Exemplos: missed [mist], passed [pæst], traveled [trævald], remembered [rimembərd], discovered [diskıvərd].

O Quadro 1, a seguir, que já faz uso da categorização apontada em (3), evidencia cada uma das formas de saída produzidas pelos aprendizes, em cada um dos momentos de coleta de dados. ${ }^{2}$

QUADRO 1 - Formas de saída produzidas pelos aprendizes

\begin{tabular}{|c|c|c|}
\hline Coletas & $\begin{array}{c}\text { Outputs } \\
\text { (Grupo A) }\end{array}$ & $\begin{array}{c}\text { Outputs } \\
\text { (Grupo B) }\end{array}$ \\
\hline $\begin{array}{l}\text { Pré-Instrução } \\
\text { (Nov/2002) }\end{array}$ & $\begin{array}{c}{[\text { livid }] \sim ~[l i v i t} \\
----\end{array}$ & $\begin{array}{c}{[\text { misid }] \sim[\text { misit }]} \\
{[\mathrm{mIst}]}\end{array}$ \\
\hline $\begin{array}{l}\text { Pós-Instrução } 1 \text { e } 2 \\
\text { (Jan e Mar/2003) }\end{array}$ & 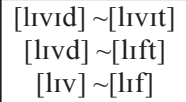 & $\begin{array}{c}\text { [mIsid] } \sim \text { misit }] \\
{[\mathrm{mIst}]} \\
{[\mathrm{mIs}]}\end{array}$ \\
\hline
\end{tabular}

É importante ressaltar, no que diz respeito aos dados do quadro em questão, que os aprendizes apresentavam todas as formas acima evidenciadas, referentes a cada um dos momentos de verificação de dados, tanto nos testes de leitura como nos de uso espontâneo da língua, não tendo havido diferenças significativas entre a modalidade de teste realizada. A observação do Quadro 1 deixa claro que, no que diz respeito a verbos do grupo $\mathrm{A}$, formas caracterizadas pela seqüência consonantal final só foram realizadas após o trabalho de instrução explícita, ou seja, a partir do segundo momento de coleta de dados. Dada essa constatação, pode-se sugerir que, com relação a esse grupo verbal, o trabalho pedagógico parece ter sido de grande importância no sentido de levar à produção das formas-alvo que ainda não eram produzidas. Chama a atenção, no que diz respeito à sonoridade, o fato de que plosivas coronais exibindo o traço [+son] já eram produzidas desde a coleta pré-instrucional, mesmo que em outputs exibindo padrões silábicos diferentes do tido como alvo (como em [livid] e [misid]).

No que diz respeito aos dados da terceira coleta, verificam-se as mesmas formas de saída obtidas na segunda, confirmando-se assim a manutenção dos efeitos do trabalho pedagógico desenvolvido. Ressalta-se a permanência, ainda assim, de variação nas formas dos aprendizes tanto no que diz respeito à produção da estrutura silábica como no que concerne à produção do traço de sonoridade das plosivas coronais finais, deixandose claro que a instrução explícita não se mostrou capaz de erradicar a produção de formas diferentes das tidas como alvo, ainda que tenha sido verificada uma diminuição dos índices de produções de formas ilícitas ao se realizar a comparação dos níveis de acuidade verificados nas segunda e terceira coletas, o que leva a sugerir uma tendência a uma futura hegemonia das formas semelhantes ao falar nativo.

\section{ANÁLISE VIA OT}

O trabalho de análise que aqui se inicia verificará individualmente os aspectos referentes à estrutura silábica e à sonoridade ${ }^{3}$. Através deste tratamento individual para cada um dos fenômenos, espera-se tornar evidente a afirmação realizada no início deste artigo: enquanto que a aquisição do traço [+son] implica apenas a demoção de restrições a partir de $\mathrm{H}_{0}$, a aquisição das codas complexas finais exige, além do processo de demoção, a aquisição de uma nova estrutura de input.

\subsection{Análise da estrutura silábica}

\subsubsection{As formas de output e suas hierarquias correspondentes}

A análise da aquisição das codas complexas fará a distinção dos verbos em grupos A e B, conforme expresso em (3). Sabendo-se que o 
aprendiz parte de um sistema hierárquico inicial que é o da L1, apresenta-se, em (4), a hierarquia referente à estrutura da sílaba do português brasileiro, tendose por base os trabalhos de Lee (1999) e Collischonn (2000).

$(4)^{4}$

Sonor, Dep ${ }^{\text {Onset }}$, Nuc, Max $>>$ CodaCond $>>$ Dep $^{\text {Nuc }}>>$ Contig $>>$ Onset $>>$ NoCoda, NoComplex

São caracterizadas, em (5), as restrições apresentadas em (4) que se mostrarão pertinentes para a presente análise (cf. Lee 1999).

(5)

Onset: Toda sílaba deve ter onset.

NoCoda: Codas são proibidas.

CodaCond: A coda pode ter somente [-vocálico, + soante] ou [soante, +contínuo, + coronal].

NoComplex: Mais de um $\mathrm{C}$ ou um $\mathrm{V}$ não podem se associar às posições da sílaba.

DEP I/O: Todos os segmentos/traços da entrada têm correspondente idêntico na saída: Dep ${ }^{\mathrm{Nuc}}$, Dep ${ }^{\text {Onset } .5}$.

MAX I/O: Todos os segmentos/traços da saída têm correspondente idêntico na entrada.

Contig: a saída é contígua à entrada.

Tem-se, no tableau em (6), a representação da hierarquia da L1, e o candidato ótimo dela decorrente, considerando-se verbos pertencentes ao grupo A. Considera-se, como representação de input, aquela apontada pela literatura da área como a tida pelos falantes nativos da língua inglesa, em que a marca de passado/particípio passado regular é caracterizada pelo segmento plosivo coronal /d/ (como em $/ \mathrm{liv}+\mathrm{d} / \mathrm{e} / \mathrm{mIs}+\mathrm{d} /$, cf. Gussenhoven \& Jacobs, 1998).

\begin{tabular}{|c|c|c|c|c|c|c|}
\hline$/ \operatorname{liv}+\mathrm{d} /$ & Max & CodaCond & Dep & Contig & NoCoda & NoComp \\
\hline a) 1 I.VI.dI & & & $* *$ & * & & 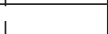 \\
\hline b) li.VI & $* !$ & & * & & & I \\
\hline c) liv.dI & & *! & * & & * & I \\
\hline d) li.vid & & *! & * & * & * & $T$ \\
\hline e) liv & *! & $*$ & & & $*$ & \\
\hline f) livd & & $* !$ & & & * & * \\
\hline
\end{tabular}

Vê-se, no tableau em (6) ${ }^{7}$, que quando aplica, para o uso da L2, a hierarquia de sua línguamãe, o aluno, no caso de codas complexas constituídas por dois segmentos não permitidos pelo português brasileiro, acaba produzindo duas sílabas do tipo CV. Veja-se, em (7), a mesma hierarquia agindo sobre candidatos cuja penúltima consoante de coda é permitida pelo português. Esses verbos apresentam, sob a hierarquia da nossa língua, apenas uma epêntese final, dado o fato de que a penúltima consoante, por ser permitida pela L1, não desrespeita a restrição CodaCond.

(7)

\begin{tabular}{|c|c|c|c|c|c|c|}
\hline$/ \mathrm{mIs}+\mathrm{d} /$ & Max & CodaCond & Dep & Contig & NoCoda & NoComp \\
\hline a) mis.di & & & * & & * & I \\
\hline b) mI.sI.dI & & & $* * !$ & $*$ & & 1 \\
\hline c) $\mathrm{mIS}$ & $* !$ & & & & * & $T$ \\
\hline d) MI.SI & $* !$ & & * & & & 1 \\
\hline e) missd & & $* !$ & $*$ & $*$ & * & 1 \\
\hline f) $\mathrm{mIst}$ & & $* !$ & & & * & \\
\hline
\end{tabular}

A partir da observação do Quadro 1, vê-se que formas como [lividi] e [misdi], os candidatos que se sagraram ótimos dos dois tableaux acima, não foram produzidos pelos aprendizes nem mesmo na coleta pré-instrucional. Isso quer dizer que, mesmo na primeira coleta de dados, os aprendizes não se encontravam mais em um estágio equivalente ao da L1, tendo já realizado demoções de restrições.

Após terem sido apresentados os outputs ótimos oriundos da hierarquia da L1, passa-se a apresentar outputs ótimos advindos de ranqueamentos de restrições diferentes dessa hierarquia inicial. Posteriormente, será discutida a questão da seqüência de aquisição de tais hierarquias.

Dando-se continuidade à tarefa de determinar a hierarquia responsável por cada uma das formas de output produzidas pelos aprendizes, vê-se que a hierarquia capaz de levar às formas-alvo é aquela em que fidelidade domina marcação, considerando como forma de input aquela em que o sufixo "-ed" se caracteriza apenas pelo segmento coronal /d/ (como em /liv $+\mathrm{d} / \mathrm{e} / \mathrm{mis}+\mathrm{d} /$ ).

$(8)^{8}$
\begin{tabular}{|l|c|c|c|c|c|c|}
\hline /liv+d/ & Max & Dep & CodaCond & Contig & NoCoda & NoComp \\
\hline a) li.vi.di & & $* ! *$ & & $*$ & & I \\
\hline b) liv.di & & $* !$ & $*$ & & $*$ & ( \\
\hline c) li.vId & & $* !$ & $*$ & $*$ & $*$ & \\
\hline d) liv & $* !$ & & $*$ & & $*$ & $*$ \\
\hline e) ${ }^{*}$ livd & & & $* *$ & & $*$ & $*$ \\
\hline
\end{tabular}

(9)

\begin{tabular}{|c|c|c|c|c|c|c|}
\hline$/ \mathrm{mIs}+\mathrm{d} /$ & Max & Dep & CodaCond & Contig & NoCoda & NoComp \\
\hline a) mis.dI & & $* !$ & & & $*$ & 1 \\
\hline b) mI.sI.dI & & $* ! *$ & & $*$ & & I \\
\hline c) mI.sId & & $* !$ & $*$ & * & $*$ & $\mathrm{I}$ \\
\hline d) mis & $* !$ & & & & * & I \\
\hline e) MI.SI & $* !$ & $*$ & & & & $T$ \\
\hline f) mist & & & $*$ & & $*$ & 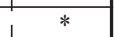 \\
\hline
\end{tabular}


Vê-se, a partir dos tableaux em (8) e (9) que, para se ter como output ótimo uma coda complexa, é preciso ter fidelidade dominando marcação. Assim, o candidato ótimo se mostra o mais fiel ao input possível. Aqui, isso significa Max e Dep dominando CodaCond.

São apresentados, também, os tableaux contendo as hierarquias responsáveis pela produção das formas que exibem, como último segmento, a consoante final da raiz verbal, como é mostrado em (10) e (11). Neste caso, a restrição Dep domina CodaCond, porém Max é dominada por esta restrição.

$(\mathbf{1 0})^{9}$
\begin{tabular}{|l|c|c|c|c|c|c|}
\hline live+d/ & Dep & CodaCond & Max & Contig & NoCoda & NoComp \\
\hline a) li.vI.dI & $* ! *$ & & & $*$ & & \\
\hline b) liv.di & $* !$ & $*$ & & & $*$ & \\
\hline c) li.vid & $* !$ & $*$ & & $*$ & $*$ & \\
\hline d) - liv & & $*$ & $*$ & & $*$ & \\
\hline e) livd & & $* * !$ & & & $*$ & $*$ \\
\hline
\end{tabular}

(11)

\begin{tabular}{|l|c|c|c|c|c|c|}
\hline mIs+d/ & Dep & CodaCond & Max & Contig & NoCoda & NoComp \\
\hline a) mIs.dI & $* !$ & & & & $*$ & \\
\hline b) mI.sI.dI & $* ! *$ & & & $*$ & & \\
\hline c) $\widetilde{\sigma}$ mIs & & & $*$ & & $*$ & \\
\hline d) mI.sId & $* !$ & $*$ & & $*$ & $*$ & \\
\hline e) mIst & & $* !$ & & & $*$ & $*$ \\
\hline
\end{tabular}

A partir dos tableaux até então apresentados, vê-se que as formas subjacentes como /liv $+\mathrm{d} /$ e / $\mathrm{mIs}+\mathrm{d} / \mathrm{se}$ mostravam como suficientemente capazes de levar à produção tanto das formas semelhantes ao alvo como das formas caracterizadas pelo apagamento do segmento plosivo coronal. Mostrarse-á, a partir de agora, que formas de output como [livid] e [misid], entretanto, não conseguem ser obtidas a partir das formas de input acima apresentadas.

\subsubsection{A coexistência de formas de input}

Ainda que seja proposta uma tentativa de se dar conta de formas de output como [livid] e [misid], caracterizadas por uma epêntese interconsonantal, a partir das restrições que compõem a hierarquia inicial e a forma de input $/ \mathrm{d} /$ , verificar-se-á que as formas de saída caracterizadas por tal padrão silábico não conseguem se sagrar como ótimas a partir da forma de input em questão.

Poder-se-ia, como uma tentativa de solução, manter-se a forma de input/d/ como aquela realmente tida pelos aprendizes, atuando-se unicamente sobre a hierarquia de restrições para se obter como ótimas as formas [livid] e [misid]. Uma possível solução seria sugerir, em princípio, que o uso da restrição $\operatorname{Son}^{10}$, proposta por Lee (1999) para a hierarquia do português, ou ainda qualquer outra restrição que se opusesse a seqüências consonantais não permitidas pela L1, poderia dar conta de tal epêntese interconsonantal. Desse modo, seqüências como [livid] e [misid] apresentariam a vogal interconsonantal para satisfazer tal restrição que estaria militando contra as seqüências consonantais proibidas pela L1, ainda que os segmentos que compunham tais seqüências já fossem produzidos individualmente, pelos aprendizes, em codas simples. Nesse sentido, ainda que CodaCond já estivesse ocupando um lugar mais baixo na hierarquia, a restrição Son, ainda estando altamente ranqueada, poderia vir a justificar as formas de output produzidas pelos aprendizes.

Caso tal possibilidade fosse realmente a explicação verdadeira para a produção de formas como [livid] e [misid], caberia discutir, ainda, a posição do segmento epentético produzido para a satisfação de Son. Uma vez que a restrição Contig, conforme pode ser visto em (4), possui caráter atuante na hierarquia do português, esperar-se-ia que o segmento epentético fosse produzido em posição final, como em [lıvdr] e [misdi], de modo a não violar tal restrição, sem mesmo assim deixar de satisfazer Son. Entretanto, não é isso que se vê nos dados: formas com segmentos epentéticos em posição final não foram produzidas em nenhum momento de coleta de dados. Seria preciso, para dar conta das formas contendo uma epêntese interconsonantal, evidenciar uma restrição que se mostrasse em posição mais alta que Contig, e que lhe fizesse oposição, para que ocorressem epênteses mediais, não finais. Retomando-se o que já foi dito, dessa forma, tal epêntese se mostraria como explicável em função da necessidade de satisfação de Son, não de CodaCond.

Não será apresentada, aqui, tal hierarquia acima sugerida, uma vez que a possibilidade de solução acima descrita parece não estar evidenciando o que realmente parece acontecer no sistema de interlíngua do aprendiz. Tal decisão sustenta-se na constatação de que, em formas não sufixadas caracterizadas por codas complexas, encerradas por um segmento outro que /S/, formas contendo uma única epêntese em posição medial, 
como aquelas identificadas em [lıvid] e [misıd], não são produzidas pelos aprendizes. Por exemplo, dada a produção de uma palavra monomorfêmica como lift /1ıft/, não se encontrará [lıfit] como forma de saída. Também em palavras como past/pæst/, que possuem a primeira consoante como permitida pelo português brasileiro, a epêntese medial mostra-se desnecessária, encontrando-se uma razão para o fato de não se encontrar, na produção dos aprendizes, formas como [pæsıt]. De fato, o que pode, sim, ser encontrado, em uma análise da produção de formas não-sufixadas do grupo A, são duas epênteses, como em [lıfitı]. No caso de verbos possuindo como penúltima consoante uma permitida em coda pelo $\mathrm{PB}$, encontra-se, sim, apenas um segmento epentético, porém em posição final, como em [pæstı].

A partir da constatação apresentada acima, questiona-se como explicar a produção de formas exibindo uma única epêntese em posição medial, como em [lıvid] e [misıd], padrão silábico esse que se mostrou predominante na primeira coleta de dados desta pesquisa, tendo havido ainda, no caso dos verbos do grupo 'A', produção unânime. As evidências acima apresentadas sugerem o fato de que formas apontando apenas uma epêntese, em posição medial, são fruto da possibilidade de uma representação subjacente, mantida pelos aprendizes, distinta daquela com que se esteve trabalhando até o presente momento. De fato, acredita-se que tal representação de input tenha surgido, prioritariamente, a partir da influência da escrita na produção de palavras com a marca de passado do inglês, representada graficamente por '-ed'. Ressalta-se o fato de que produções exibindo padrões silábicos como os de [lıvid] e [misıd] só eram encontradas em tentativas de produção de verbos contendo a marca "-ed”. Desse modo, uma explicação para a questão encontra-se na possibilidade de os alunos possuírem, como representação de input de tais verbos, formas como $/ 1 \mathrm{IV}+\mathrm{Id} / \mathrm{e} / \mathrm{mIs}+\mathrm{Id} /$, contendo, na própria forma subjacente, um segmento vocálico. Assim, formas, pertencentes ao grupo 'B', com produção semelhante àquela em [misid], que apresentariam, sob a forma subjacente $/ \mathrm{mIs}+\mathrm{d} /$, uma epêntese desnecessária, uma vez que tal segmento vocálico segue um elemento permitido na coda do português, são abarcadas pela presente solução.

Como comprovação do que foi até o presente momento afirmado, os tableaux a seguir têm o objetivo de evidenciar a hierarquia de restrições para as formas [livid] e [misid] a partir de formas de input que contenham, também, um segmento vocálico que inviabiliza a formação de uma coda complexa - ressalta-se novamente que a presença desse segmento na representação subjacente se mostra decorrente da grafia das formas verbais, que exibem o grafema ' $\mathrm{e}$ '.

(12)

\begin{tabular}{|l|c|c|c|c|c|c|}
\hline liv+Id/ & Max & Dep & CodaCond & Contig & NoCoda & NoComp \\
\hline a) li.vi.di & & $* !$ & & & & I \\
\hline b) liv.di & $* !$ & $*$ & $*$ & $*$ & $*$ & I \\
\hline c) - II.VId & & & $*$ & & $*$ & \\
\hline d) liv & $* ! *$ & & $*$ & & $*$ & \\
\hline e) livd & $* !$ & & $* *$ & $*$ & $*$ & $*$ \\
\hline
\end{tabular}

(13)

\begin{tabular}{|c|c|c|c|c|c|c|}
\hline$/ \mathrm{mIS}+\mathrm{Id} /$ & $\operatorname{Max}$ & Dep & CodaCond & Contig & NoCoda & ${ }^{\top}$ NoComp \\
\hline a) mIs.dI & $* !$ & * & & * & * & I \\
\hline b) mI.SI.dI & & $* !$ & & & & 1 \\
\hline c) MI.SI & $* !$ & & & & & 1 \\
\hline d) ${ }^{2}$ I.SId & & & * & & * & 1 \\
\hline e) $\mathrm{mIS}$ & $* ! *$ & & & & * & \\
\hline f) misd & $* !$ & & * & * & $*$ & * \\
\hline
\end{tabular}

Vê-se que, a partir do input $/ 1 \mathrm{IV}+\mathrm{Id} /$, tem-se o output ótimo [livid] como advindo de uma hierarquia que se diferencia de $\mathrm{H}_{0}$ unicamente pelo fato de já ter realizado a demoção de CodaCond. Tal forma subjacente, além disso, também consegue prover uma explicação mais satisfatória para formas como [misid], cujo [I], sob o input $/ \mathrm{mIs}+\mathrm{d} /$, mostrava-se como injustificável pelo fato de ser um segmento epentético advindo de uma consoante permitida em coda pela L1. A adoção de /mIs+Id/ como input, dessa forma, explica melhor a natureza de tal segmento vocálico, parecendo ser, portanto, a solução apropriada para a manifestação das formas de output do grupo B que contenham apenas uma epêntese em posição medial.

Cabe, a partir dessas constatações, concluir a discussão acerca da pertinência ou não, para fins deste estudo, de uma restrição como Son, que se oponha às seqüências consonantais não permitidas pela L1. Uma vez que formas exibindo apenas uma epêntese em posição final, como [lıvdi] e [misdi], não foram encontradas nos dados desta pesquisa, e outputs como [livid] e [misid] mostram-se, conforme foi apontado pelos tableaux acima, decorrentes das formas de input / $1 \mathrm{IV}+\mathrm{Id} /$ e /mIs+Id/, não é necessário contar, para a explicação dos dados aqui obtidos, com tal restrição assumindo papel 
algum de decisão, deixando-se, no que diz respeito aos dados aqui verificados, a responsabilidade pelo surgimento de segmentos epentéticos unicamente à restrição CodaCond. Vê-se, de fato, que a invocação de tal restrição não se mostra necessária para fins desta análise. Deve-se indagar, conforme já anteriormente dito, a respeito da pertinência de tal restrição em outros estudos da aquisição de codas complexas por falantes do português brasileiro. Deixa-se, assim, a questão em aberto, invocandose a necessidade de dados de aquisição que evidenciem manifestações, tanto em formas sufixadas ou não, como lift [1If.tr] e lived [1Iv.di], em que se prove que, em codas simples, tais plosivas coronais finais já sejam produzidas. Crê-se que, somente após tal constatação, se mostrará clara a necessidade da proposta de uma restrição que se oponha especificamente a seqüências de consoantes em coda, nesse caso as não-permitidas pela L1, restrição essa que no caso em questão se encontraria em uma posição alta na hierarquia, ainda que CodaCond já tivesse sido demovida para uma posição mais baixa no ranking. Caso seja evidenciada a necessidade de uma restrição dessa natureza, estar-se-á deixando claro o fato de que a epêntese pode ter não somente a função, conforme já foi dito, de satisfazer CodaCond, mas também o papel de satisfazer restrições que se oponham a seqüências não permitidas em coda, ainda que cada um dos segmentos que componham tal seqüência já sejam produzidos corretamente em codas simples. Por ora, isso é o máximo que se pode dizer acerca da questão.

Evidenciou-se, assim, que as formas de output produzidas pelos sujeitos deste estudo se mostram decorrentes de duas formas de input distintas. Formas como [livid] e [misid], conforme já evidenciado, advêm de estruturas subjacentes originárias da escrita, como / $1 \mathrm{Iv}+\mathrm{Id} / \mathrm{e} / \mathrm{mIs}+\mathrm{Id} /$. Já quando os alunos produziam a forma-alvo, ou formas encerradas pelo segmento final da raiz, tais outputs se davam em função de uma estrutura de input do tipo $/ \mathrm{liv}+\mathrm{d} / \mathrm{e} / \mathrm{mIs}+\mathrm{d} /$. Nesse sentido, fica claro o fato de que, para ser capaz de produzir as formas semelhantes ao falar nativo, é preciso que o aprendiz abandone aquela representação de input advinda da escrita, adquirindo, assim, formas de input como $/ \mathrm{l} \mathrm{Iv}+\mathrm{d} / \mathrm{e} / \mathrm{mIs}+\mathrm{d} /$, tarefa essa que se acredita ter sido facilitada pelo trabalho de instrução explícita.

\subsubsection{A seqüência de aquisição da estrutura silábica}

Tendo-se determinado as hierarquias e os inputs responsáveis por cada uma das formas de output produzidas pelos aprendizes, passa-se, a partir desse momento, para a verificação da seqüência de aquisição da estrutura silábica dos sujeitos pesquisados, tomando-se por base o algoritmo de Tesar \& Smolensky $(1996,2000)$.

A observação dos dados referentes ao período pré-instrucional, verificáveis no Quadro 1, aponta para o fato de que os alunos não apresentam mais a hierarquia do português. Uma vez que formas pertencentes ao grupo A, exibindo epêntese medial, se mostravam como unânimes nesse estágio, conclui-se que a restrição CodaCond já havia sido, previamente, demovida pelos aprendizes, de modo que os aprendizes exibam a hierarquia Max $>>$ Dep $>>$ CodaCond. A forma subjacente em jogo é aquela contendo o segmento vocálico. Formas decorrentes do input $/ 1 \mathrm{Iv}+\mathrm{d} /$ serão produzidas, conforme pode ser visto também no Quadro 1, somente após a realização do trabalho de instrução explícita. No caso dos verbos pertencentes ao grupo $\mathrm{B}$, já há, desde a etapa pré instrucional, uma variação entre formas de saída contendo a epêntese interconsonantal e encontros consonantais bem formados em coda, semelhantes àqueles produzidos por falantes nativos. Tal variação não decorre da reorganização de restrições, uma vez que a relação hierárquica em jogo é, também, aquela em que Max $>>$ Dep $>>$ CodaCond. De fato, tal variação, entre formas semelhantes ao alvo ou não, se dá em função da forma subjacente, capaz de ocasionar outputs distintos.

Em suma, é apresentada, em (14), a hierarquia que caracteriza o estágio de interlíngua em que se encontravam os aprendizes antes da instrução explícita, durante a primeira coleta de dados. Reconhece-se, no caso dos verbos do grupo $\mathrm{B}$, a variabilidade no que diz respeito às formas de output em função da coexistência de duas formas subjacentes para a marca de passado ${ }^{11}$.

(14)

Hierarquia do Período Pré-Instrucional ( $1^{\mathrm{a}}$ coleta - nov/2002) Max $>>$ Dep $>>$ CodaCond $^{12}$

Passa-se a analisar o estágio de aquisição em que se encontravam os aprendizes no período da segunda coleta de dados, a primeira verificação após 
a realização do trabalho de instrução explícita.

Os dados referentes à produção da estrutura silábica na primeira coleta após a instrução explícita indicam que já podia ser verificada a coexistência de formas subjacentes tanto no grupo B, como também no grupo A. Sugere-se, nesse sentido, que a instrução explícita colaborou com o surgimento de uma nova estrutura de input, no que diz respeito a verbos como lived. Além da coexistência das duas formas subjacentes relativas a cada um dos grupos, que originam a variação entre formas de output contendo uma coda complexa ou um segmento vocálico entre as duas consoantes que comporiam tal coda, há, também, manifestações variáveis caracterizadas por codas simples, encerradas pelo último segmento consonantal que compõe a raiz de tais verbos.

Para dar conta da variação entre as formas exibindo apagamento da plosiva final e as tidas como alvo, ambas decorrentes da mesma forma subjacente, fez-se uso da proposta de estratos hierarquizados de Bonilha \& Matzenauer (2003), proposta essa que se fundamenta sobre o pressuposto de que há uma relação hierárquica dentro de um estrato que compartilha restrições. A variação, nesse sentido, se mostra explicável uma vez que se considere a possibilidade de as restrições que compõem tais estratos apresentarem um ranqueamento 'flutuante' entre si.

Assim, quando, dentro do estrato, a relação hierárquica estabelecida é $\{$ Max $>$ CodaCond $\}$, terse-á como output ótimo a forma-alvo (tableaux em 15 e 17), e quando a relação hierárquica estabelecida é $\{$ CodaCond $>>$ Max $\}$, a forma de saída será a que exibe apagamento da plosiva coronal final (tableaux em 16 e 18).

\section{Grupo 'A'}

(15)

\begin{tabular}{|c|c|c|c|c|}
\hline /liv+d/ & Dep & Max CodaCond & Contig & NoCoda NoComp \\
\hline li.VI.di & $* ! *$ & 1 & * & \\
\hline II.VI & $* !$ & $*$ & & 1 \\
\hline livid & $* !$ & $*$ & * & $*$ \\
\hline liv & & $* ! \quad 1$ & & * \\
\hline livd & & $* *$ & & * \\
\hline
\end{tabular}

\section{(16)}

\begin{tabular}{|l|c|c|c|c|c|c|}
\hline liv+d/ & Dep & CodaCond & Max & Contig & NoCoda & NoComp \\
\hline a) li.vi.dI & $* ! *$ & & & $*$ & & । \\
\hline b) li.vi & $* !$ & & $*$ & & & । \\
\hline c) li.vid & $* !$ & $*$ & & $*$ & $*$ & $*$ \\
\hline d) liv & & $*$ & $*$ & & $*$ & $*$ \\
\hline e) livd & & $* * !$ & & & $*$ & $*$ \\
\hline
\end{tabular}

$\underline{\text { Grupo 'B' }}$

(17)

\begin{tabular}{|c|c|c|c|c|}
\hline$/ \mathrm{mIs}+\mathrm{d} /$ & Dep & Max CodaCond & Contig & NoCoda ${ }^{\prime}$ NoComp \\
\hline a) mIs.dI & $* !$ & 1 & & \begin{tabular}{|l|l|}
$*$ & 1 \\
\end{tabular} \\
\hline b) mI.SI.dI & $* ! *$ & 1 & * & 1 \\
\hline c) $\mathrm{mIS}$ & & $* !$ & & $*$ \\
\hline d) $\mathrm{mI} . \mathrm{SI}$ & $* !$ & $* 1$ & & $T$ \\
\hline e) mi.sid & $* !$ & $*$ & $*$ & * \\
\hline f) mIst & & * & & * \\
\hline
\end{tabular}

(18)

\begin{tabular}{|c|c|c|c|c|c|}
\hline$/ \mathrm{mIs}+\mathrm{d} /$ & Dep & CodaCond'Max & Contig & NoCoda & NoComp \\
\hline a) mIs.di & $* !$ & 1 & & * & 1 \\
\hline b) mI.SI.dI & $* ! *$ & 1 & * & & 1 \\
\hline c) $\mathrm{mIs}$ & & $1 *$ & & * & 1 \\
\hline d) MI.SI & $* !$ & $1 *$ & & & $T$ \\
\hline e) missid & $* !$ & * & $*$ & * & \\
\hline f) mist & & *! & & $*$ & \\
\hline
\end{tabular}

É importante ressaltar, ainda, que formas exibindo uma única epêntese em posição medial, decorrentes de inputs do tipo /liv $+\mathrm{Id} / \mathrm{e} / \mathrm{mIs}+\mathrm{Id} /$, se mostram garantidas através da mesma hierarquia, desde que respeitada a exigência de que o estrato exiba Max como restrição dominante. Assim, a hierarquia de restrições que justifica as formas de output encontradas no segundo momento de verificação de dados, a primeira coleta após a instrução explícita, é a apresentada em (19). Essa, também, é a hierarquia correspondente ao terceiro momento de coleta de dados (segunda coleta pós-instrucional), uma vez que os padrões silábicos nas formas de saída produzidas nas duas coletas pós-instrucionais, conforme pode ser visto no Quadro 1, são os mesmos.

(19)

Hierarquia Pós-Instrucional 1 e 2 (jan e mar/2003)

Dep>>Max,CodaCond ${ }^{13}$

Em resumo, o Quadro 2 apresenta as hierarquias, referentes à aquisição da estrutura silábica, características de cada uma das três coletas de dados, bem como as formas subjacente e de output presentes em cada uma dessas coletas:

Quadro 2 - Seqüência de aquisição da estrutura silábica ${ }^{14}$

\begin{tabular}{|c|c|c|c|c|}
\hline Hierarquia & Input (A) & Output $(\mathrm{A})$ & Input (B) & Output (B) \\
\hline $\begin{array}{l}\text { Pré-Instrução } \\
\text { Max }>>\text { Dep }>>\text { CodaCond }\end{array}$ & $\begin{array}{r}/ \mathrm{liv}+\mathrm{Id} / \\
\quad 15\end{array}$ & [livid] & $\begin{array}{c}/ \mathrm{mIs}+\mathrm{Id} / \\
/ \mathrm{mIs}+\mathrm{d} /\end{array}$ & $\begin{array}{c}{[\mathrm{mIsid}]} \\
{[\mathrm{mIst}]}\end{array}$ \\
\hline $\begin{array}{l}\text { Pós-Instrução 1(Jan) } \\
\text { Dep >> Max,CodaCond }\end{array}$ & $\begin{array}{r}/ \mathrm{liv}+\mathrm{Id} / \\
\text { lliv }_{-}+\mathrm{d} /\end{array}$ & $\begin{array}{l}\text { [livid] } \\
{[\text { livd] }}\end{array}$ & $\begin{array}{c}/ \mathrm{mis}+\mathrm{Id} / \\
/ \mathrm{mIS}+\mathrm{d} /\end{array}$ & $\begin{array}{l}{[\mathrm{misid}]} \\
{[\mathrm{mIst}]}\end{array}$ \\
\hline & $/ \mathrm{liv}+\mathrm{d} /$ & [liv $]$ & $/ \mathrm{mIs}+\mathrm{d} /$ & [mIs] \\
\hline
\end{tabular}

ORGANON PORTO ALEGRE v.18 n. 36 p. 57-71 2004 


\begin{tabular}{|c|c|c|c|c|}
\hline & & & & $\begin{array}{l}\text { tinuação } \\
\text { o quadro } \\
\text { anterior }\end{array}$ \\
\hline $\begin{array}{l}\text { Pós-Instrução } 2 \text { (Mar) } \\
\text { Dep >> Max,CodaCond }\end{array}$ & $\begin{array}{l}/ \mathrm{liv}+\mathrm{Id} / \\
/ \mathrm{liv}+\mathrm{d} /\end{array}$ & $\begin{array}{l}\text { [livid] } \\
\text { [livd] }\end{array}$ & $\begin{array}{l}/ \mathrm{mIs}+\mathrm{Id} / \\
/ \mathrm{mIS}+\mathrm{d} /\end{array}$ & $\begin{array}{c}{[\mathrm{misId}]} \\
{[\mathrm{mIst}]}\end{array}$ \\
\hline Dep $\gg \overline{\text { CodaCond,Max }} \overline{-}-\bar{c}$ & $\overline{\mathrm{livd} /}$ & $\frac{--}{[\mathrm{liv}]}$ & $\overline{/ \mathrm{mIsd} /}$ & $\overline{[\mathrm{mIs}]}$ \\
\hline
\end{tabular}

Deve-se considerar, conforme foi afirmado nos parágrafos acima, a possibilidade de "flutuação" das restrições internas aos estratos e de a relação entre essas restrições implicar dominância, o que justificará as produções variáveis das formas de output. As hierarquias apresentadas no quadro acima confirmam a capacidade, por parte da instrução explícita, sobretudo, de instaurar e fortalecer, entre os aprendizes, uma outra representação subjacente acerca dos verbos contendo o morfema de passado/ particípio passado simples do inglês, input esse capaz de levar à produção das formas-alvo e que, pelo menos até a última coleta de dados realizada, se mostra em variação com a forma de input anteriormente mantida pelos aprendizes. Pelo fato de as manifestações oriundas do input primeiramente estabelecido se mostrarem cada vez menores, é possível que tenha continuado a haver, em períodos posteriores à última coleta, a gradual diminuição de formas de output oriundas de tal input de caráter indevido, caracterizando a aquisição, desse modo, como um estágio dinâmico, ainda em andamento.

Confirma-se, assim, a dupla tarefa do aprendiz, no que diz respeito à aquisição das estruturas silábicas dos verbos contendo o sufixo "-ed" : demoção de restrições e aquisição de um novo input, input esse diferente daquele instaurado pela forma grafada. A seguir, tratar-se-á da questão da sonoridade do segmento plosiva final, de modo a se evidenciar a tarefa única do aprendiz, conforme já apontado: a demoção de restrições.

\subsection{Análise da sonoridade da plosiva coronal final}

\subsubsection{As formas de output e suas hierarquias correspondentes}

Na pesquisa, mostrou-se necessário realizar, também, a distinção dos verbos em categorias, de acordo com a sonoridade do segmento coronal final, categorização essa apresentada em (20):
(20)

Grupo 1 - /d/ produzido como [d] em função de a consoante que o antecede possuir o traço [+son]. Exemplos: lived

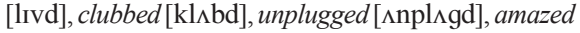
[əmeIzd], absorbed [əbsorbd], traveled [trævəld], remembered [rimembərd], turned [t3:rnd].

Grupo 2 - /d/ produzido como [t] em função de a consoante que $\mathrm{o}$ antecede possuir o traço [-son]. Exemplos: missed [mist], attacked [ətækt], stopped [sta:pt], rushed [r $\wedge \mathrm{t}$ ], laughed [læft], watched [wa:t $\mathrm{t}]$, asked [æskt].

São apresentadas, em (21), as restrições utilizadas na análise:

(21)

Agree: Encontros de obstruintes concordam no que diz respeito ao vozeamento.

] $\sigma / *$ Voice: Obstruintes sonoras em posição final de sílaba são proibidas.

Ident(Voice): O segmento de output deve se mostrar idêntico, em termos de vozeamento, ao seu segmento de input correspondente (cf. McCarthy 2002) ${ }^{16}$.

No que diz respeito a estruturas silábicas compostas por uma coda complexa, tem-se na hierarquia Agree $>>\operatorname{Ident}($ Voice $)>>\sigma / *$ Voice aquela capaz de refletir os efeitos da assimilação progressiva de sonoridade relativa ao passado dos verbos regulares. Os tableaux em (22) e (23) evidenciam o acima afirmado. ${ }^{17}$

(22)

\begin{tabular}{|l|c|c|c|}
\hline$/$ liv $+\mathrm{d} /$ & Agree & Ident(Voice) & ] $/{ }^{*}$ voice \\
\hline a) ${ }^{*} \operatorname{livd}$ & & & $* *$ \\
\hline b) lift & & ${ }^{*}$ & \\
\hline c) livt & $* !$ & ${ }^{*}$ & $*$ \\
\hline d) lifd & $* !$ & ${ }^{*}$ & ${ }^{*}$ \\
\hline
\end{tabular}

(23)

\begin{tabular}{|c|c|c|c|}
\hline$/ \mathrm{mIs}+\mathrm{d} /$ & Agree & Ident(Voice) & ]$\sigma / *$ voice \\
\hline a) mizd & & $*$ & $* ! *$ \\
\hline b) $\mathrm{mIst}$ & & * & \\
\hline c) mIzt & $* !$ & $* *$ & * \\
\hline d) misd & $* !$ & & * \\
\hline
\end{tabular}

Para a representação de dessonorizações inadequadas, basta inverter a relação de dominância entre as restrições $] \sigma / *$ voice e Ident (Voice), como mostra o tableau em (24) ${ }^{18}$.

(24)

\begin{tabular}{|l|c|c|c|}
\hline$/$ liv $+d /$ & Agree & ] $\sigma / *$ voice & Ident(Voice) \\
\hline a) livd & & $* ! *$ & \\
\hline b) $\operatorname{\sigma r}$ lift & & & $* *$ \\
\hline c) livt & $* !$ & $*$ & $*$ \\
\hline d) lifd & $* !$ & $*$ & $*$ \\
\hline
\end{tabular}


Vê-se que também com a relação ] $\sigma / *$ voice >> Ident(voice), tem-se, no caso dos verbos pertencentes ao grupo 2, a plosiva coronal exibindo o traço [-son]. Independentemente da relação de dominância entre essas duas restrições, a decisão cai sobre $] \sigma /{ }^{*}$ voice, o que leva a se ter sempre o segmento final exibindo o traço [-son]. Isso foi confirmado pelos dados, pois, conforme pode ser visto no Quadro 1, não foram encontradas manifestações de verbos do grupo B produzidos com a plosiva coronal final exibindo o traço [+son], o que pode ser verificado através da observação dos tableaux em (25) e (26).

(25)

\begin{tabular}{|l|c|c|c|}
\hline$/ \mathrm{mIs}+\mathrm{d} /$ & Agree & ]$\sigma /{ }^{*}$ voice & Ident(Voice) \\
\hline a) mIzd & & $* ! *$ & $*$ \\
\hline b) $\sigma^{*} \mathrm{mist}$ & & & $*$ \\
\hline c) mIzt & $* !$ & $*$ & $* *$ \\
\hline d) mIsd & $* !$ & $*$ & \\
\hline
\end{tabular}

(26)

\begin{tabular}{|l|c|c|c|}
\hline$/ \mathrm{mIs}+\mathrm{d} /$ & Agree & Ident(Voice) & ]$\sigma /{ }^{*}$ voice \\
\hline a) mIzd & & $*$ & $* ! *$ \\
\hline b) $\varpi \mathrm{mISt}$ & & ${ }^{*}$ & \\
\hline c) mIzt & $* !$ & & $*$ \\
\hline d) $\mathrm{mIsd}$ & $* !$ & & $*$ \\
\hline
\end{tabular}

Poder-se-ia perguntar se, realmente, /d/, e não /t/, caracteriza a representação subjacente dos aprendizes. A evidência da escrita, que, no que diz respeito à estrutura silábica, foi capaz de levar a formas subjacentes de caráter inadequado do tipo / $\mathrm{IIV}+\mathrm{Id} / \mathrm{e} / \mathrm{mIS}+\mathrm{Id} /$, constitui um forte argumento para que se mantenha a posição de ser a plosiva final exibindo o traço positivo de sonoridade, realmente, a representação mantida pelos aprendizes, uma vez que a marca de passado simples/particípio passado do inglês é grafada como " ed", e não " et" . Além disso, caso os aprendizes tivessem /t/ como input, candidatos pertencentes ao grupo 1 como [livd], exibindo um encontro consonantal final sonoro, nunca se sagrariam como ótimos, independentemente da relação hierárquica entre ] $\sigma / *$ voice e Ident(Voice). Isso fica claro nos tableaux em (27) e (28).

(27)

\begin{tabular}{|l|c|c|c|}
\hline$/$ liv $+d /$ & Agree & ]$\sigma / *$ voice & Ident(Voice) \\
\hline a) livd & & $* ! *$ & $*$ \\
\hline b) ${ }^{*}$ lift & & & $*$ \\
\hline c) livt & $* !$ & $*$ & $* *$ \\
\hline d) lifd & $* !$ & $*$ & $* *$ \\
\hline
\end{tabular}

(28)

\begin{tabular}{|l|c|c|c|}
\hline$/$ liv $+\mathrm{d} /$ & Agree & Ident(Voice) & ]$\sigma /{ }^{*}$ voice \\
\hline a) livd & & $*$ & $* !$ \\
\hline b) - lift & & $*$ & \\
\hline c) livt & $* !$ & & $*$ \\
\hline d) lifd & $* !$ & $* *$ & $*$ \\
\hline
\end{tabular}

As três restrições aqui apresentadas se organizam, nos tableaux em (27) e (28), de modo a exibir os efeitos de uma assimilação de sonoridade em que, sempre que houver discrepância de sonoridade nos segmentos que compõem a seqüência consonantal final do input, o candidato ótimo será aquele que contiver o encontro consonantal exibindo o traço [-son], independentemente do ranqueamento das restrições Ident (Voice) e ] $\sigma /{ }^{*}$ voice. A análise desenvolvida de (22) a (26), dessa forma, parece ser apropriada não somente pelo fato de não permitir que candidatos do grupo 2 se sagrem como ótimos exibindo o valor [+son], o que foi confirmado pelos dados, mas também por evidenciar que, realmente, é positivo o valor do traço de sonoridade da plosiva coronal do input, em consonância com o que diz a literatura da área ao se referir ao valor de sonoridade da plosiva coronal do input mantido pelos falantes nativos da língua (cf. Gussenhoven \& Jacobs, 1998).

Vê-se, assim, que a capacidade de produção de plosivas com o traço [+son] não implica o abandono de uma representação subjacente de caráter inadequado (como, por exemplo, inputs hipotéticos do tipo/t/ ou /It/) no que diz respeito à sonoridade. Cabe aos aprendizes, conforme afirmado logo no início do texto, somente a demoção de restrições a partir da hierarquia da L1. Em outras palavras, confirma-se que a forma de input mantida pelos aprendizes, desde os períodos iniciais da aquisição, se mostrava adequada no que diz respeito à sonoridade do segmento plosivo final.

\subsubsection{A seqüência de aquisição da sonoridade}

Mesmo que, já no primeiro momento de coleta, os aprendizes em questão não mais exibam a hierarquia da L1, pois predominante já era o número de ocorrências de plosivas finais exibindo o valor positivo do traço de sonoridade, é preciso fazer considerações a respeito da posição das três restrições em questão no sistema do português brasileiro $\left(\mathrm{H}_{0}\right)$.

Acredita-se que, pelo fato de o português não 
exibir seqüências de obstruintes, Agree se encontra em uma posição não-dominada nos sistemas de L1 desses aprendizes, uma vez que nunca precisou ser demovida. No que diz respeito ao posicionamento de $] \sigma / *$ voice e Ident (voice) na hierarquia da L1, considera-se que a relação hierárquica seja aquela em que a restrição que se opõe a obstruintes sonoras em coda domine a restrição de fidelidade. A relação hierárquica entre essas duas restrições, no sistema do português brasileiro, pode ser demonstrada ao se considerar a questão da sonoridade da fricativa alveolar em final de sílabas, responsável pela variação entre a formas surda $[\mathrm{s}]$ e sonora $[\mathrm{z}] \mathrm{em}$ função do contexto fonológico seguinte. Seguindose Lopez (1979, p. 117, 137), assumir-se-á, para fins desta análise, a fricativa final como decorrente da forma subjacente /z/. Considerando-se/z/ no input, tem-se que, segundo a hierarquia que será aqui proposta, o [z] de uma palavra como vozear, em onset, mostra-se como fiel ao input [+son]. Já em uma palavra como $v o z$, em que a fricativa se encontra na posição de coda, são sentidos os efeitos de ] $\sigma /$ *voice, que não permite obstruintes sonoras em coda, e que, portanto, se encontra dominando Ident(Voice). Tem-se, assim, a mesma relação encontrada no alemão: obstruintes sonoras são proibidas em posição final ${ }^{19}$, mas em onsets são, sim, permitidos. Nesse sentido, pode-se sugerir que, no português, a restrição que se opõe a obstruintes vozeadas em coda deve dominar a de identidade.

Pode-se sugerir que a hierarquia da L1 possui a seguinte relação de dominância, apresentada em (29).

\section{(29)}

Hierarquia da L1

Agree $>>\mid \sigma / *$ Voice $>>$ Ident(Voice)

Partindo-se da idéia de ser (29) a hierarquia da L1, vê-se que, no que diz respeito à questão da sonoridade, o papel da instrução explícita seria o de demover a restrição $] \sigma / *$ Voice, de modo que Ident(Voice) a dominasse, para se terem os efeitos de assimilação de sonoridade apropriados. Entretanto, o que se pôde ver, nos dados desta pesquisa, foi uma ocorrência variável no que diz respeito à sonoridade da plosiva final. A análise individual de cada um dos momentos de coleta deixará a situação mais clara.

No que diz respeito aos dados da coleta préinstrucional, já se faz perceptível uma produção variável no que diz respeito à sonoridade da plosiva final. Tal variabilidade se mostra explicável através do ranqueamento interno das restrições que compõem um único estrato em que se encontram ] $\sigma / *$ e Ident(Voice), como se vê em (30) e (31).

(30)

\begin{tabular}{|l|c|c|c|}
\hline$/$ liv $+d /$ & Agree & Ident(Voice) & ] $\sigma^{*}$ voice \\
\hline a) $\operatorname{livd}$ & & & $* *$ \\
\hline b) lift & & $* ! *$ & \\
\hline c) livt & $* !$ & ${ }^{*}$ & $*$ \\
\hline d) lifd & $* !$ & ${ }^{*}$ & ${ }^{*}$ \\
\hline
\end{tabular}

(31)

\begin{tabular}{|l|c|c|c|}
\hline$/$ IIv $+d /$ & Agree & ]$\sigma / *$ voice & Ident(Voice) \\
\hline a) livd & & $* ! *$ & \\
\hline b) $\operatorname{\sigma } \operatorname{lift}$ & & & ${ }^{*}$ \\
\hline c) livt & $* !$ & ${ }^{*}$ & ${ }^{*}$ \\
\hline d) lifd & $* !$ & ${ }^{*}$ & ${ }^{*}$ \\
\hline
\end{tabular}

Mesmo antes da realização da instrução explícita, os dados mostram que a restrição que milita contra obstruintes sonoras em coda já havia sido demovida da hierarquia da L1, passando a pertencer ao mesmo estrato de Ident(Voice). Reconhece-se, já nesse estágio, que a relação hierárquica em que Ident(Voice) domina ] $\sigma / *$ voice se mostra como a predominante dentro do estrato. Em suma, no período pré-instrucional contava-se com a seguinte hierarquia de restrições, apresentada em (32):

\section{(32)}

Hierarquia, referente à sonoridade, no período pré-instrucional Agree $>>$ Ident(Voice), ] $\sigma / *$ Voice

A variação referente à sonoridade da plosiva coronal final se mantém mesmo após a instrução explícita, em ambas as coletas realizadas posteriormente ao trabalho pedagógico desenvolvido.

Assim, a hierarquia apresentada em (33), referente ao período pós-instrucional, é a mesma representativa dos meses de janeiro e março, as duas coletas posteriores à instrução explícita.

(33)

Hierarquia, referente à sonoridade, no período pós-instrucional Agree $>>$ Ident(Voice), ] $\sigma / *$ Voice

A produção hegemônica do traço [+son] da plosiva coronal final, ainda que o aprendiz não tenha 
chegado a esse estágio, implicaria apenas a demoção da restrição ] $\sigma / *$ Voice que se mostra pertencente ao estrato hierarquizado, de modo a se ter o estágio final Ident(Voice) $>>$ ] $\sigma / *$ Voice. Reafirma-se, assim, que a aquisição do valor positivo de vozeamento do segmento /d/ final requer, apenas, tal demoção de restrições, de modo que as formas de input mantidas pelos aprendizes não representem empecilho na produção de [d] em coda, não havendo necessidade, portanto, de abandono de uma determinada forma de input por outra.

\section{CONCLUSÃO}

O presente trabalho mostrou, através da análise realizada, a capacidade da OT de demonstrar os diversos níveis de interlíngua por que passam os aprendizes, através dos diferentes ranqueamentos de restrições universais, tendo sido utilizado, para isso, o algoritmo de aprendizagem de Tesar \& Smolensky (1996, 2000), segundo o qual depreendese que adquirir uma segunda língua é demover restrições a partir do sistema da L1. Além disso, a análise desenvolvida mostrou-se de importância pelo fato de demonstrar que formas da L2, muitas vezes, são atingidas não somente através da demoção de restrições, mas também por implicarem o abandono de uma determinada representação subjacente e a subseqüente aquisição de uma nova forma de input.

A análise individual das questões da sílaba e da sonoridade, referentes à marca de passado simples/ particípio passado "-ed" do inglês, demonstrou o acima afirmado. Ainda que a demoção de restrições, a partir de $\mathrm{H}_{0}$, seja indiscutivelmente necessária para a aquisição de estruturas semelhantes ao alvo tanto no que diz respeito à questão da sílaba quanto à sonoridade, viu-se que, no caso das estruturas silábicas, cabia ao aprendiz, também, abandonar o input /Id/ decorrente da escrita, de modo a adquirir uma representação subjacente /d/, a única, conforme os tableaux apresentados, capaz de levar à produção de seqüências consonantais finais em coda.

Acredita-se que, no caso dos sujeitos da presente pesquisa, a aquisição dessas novas formas subjacentes foi facilitada por um trabalho de instrução formal acerca da produção das formasalvo, a partir do qual os aprendizes possivelmente notaram mais atentamente os detalhes da forma a que eram expostos. É interessante chamar a atenção para o fato de que, já no período da primeira coleta de dados, anterior mesmo ao trabalho de instrução formal, os alunos já haviam realizado demoções de restrições capazes de levá-los a hierarquias a partir das quais já resultariam outputs ótimos semelhantes ao falar nativo: o que faltava, realmente, era a forma subjacente adequada, conforme pôde ser visto nos dados de produções dos verbos pertencentes ao grupo A.

Ressalta-se, a partir da observação dos outputs produzidos pelos aprendizes e da análise realizada, que a variação nas formas de output, no que diz respeito à estrutura silábica, dava-se não somente em função da flutuação das restrições que compunham o estrato hierarquizado, mas também em função da própria co-ocorrência de formas de input. Tal variabilidade, decorrente tanto da hierarquia de restrições como do input, mostrou-se presente até a última coleta de dados, de modo que não se possa atribuir à instrução explícita efeitos de caráter imediato no sentido de demover restrições a ponto de desfazer estratos hierarquizados, nem de sagrar a estrutura de input recém-adquirida como hegemônica entre os aprendizes. Acredita-se, dada a verificação de um aumento nas produções semelhantes às formas-alvo, ao se fazer uma comparação dos índices numéricos dos dois últimos momentos de coleta de dados, haver uma tendência de aumento no nível de acuidade das produções, o que leva a acreditar que, posteriormente, os aprendizes abandonem a forma de input de caráter inadequado, bem como continuem a demover restrições de modo a desfazer os estratos responsáveis pela variação.

Para o pesquisador que trabalha com a questão da aquisição fonológica de L2, esta pesquisa revelou questões importantes que podem contribuir com os estudos vindouros, relativos, sobretudo, à questão da aquisição silábica do inglês como L2. Uma vez que foi verificado que a não-produção das codas complexas por parte dos aprendizes, no período pré-instrucional, não se deu devido ao fato de as suas gramáticas não permitirem a produção de seqüências finais de consoantes, mas, sim, em função da estrutura subjacente que tais alunos mantinham, é preciso fazer a distinção, nos estudos preocupados em pesquisar a produção da estrutura silábica, entre a não-aquisição da hierarquia capaz de sagrar a estrutura silábica da L2 como ótima e a não-aquisição da representação subjacente referente à estrutura silábica cuja aquisição está sendo estudada. Estudos preocupados unicamente em 
investigar a gramática dos aprendizes, referentes à questão da estrutura silábica, devem ter o cuidado, em seus trabalhos de coleta e descrição de dados, de avaliar apenas exemplos de palavras que sejam caracterizadas por seqüências consonantais também na própria forma escrita.

Assim, o trabalho que aqui se encerra, além de ter reafirmado o poder da Teoria da Otimidade de lidar com a variabilidade e o progresso na língua do aprendiz através do jogo na hierarquia de restrições, introduz uma nova questão: formas de output variáveis podem ser decorrentes, no caso de aquisição de segunda língua, de mais de uma representação subjacente por parte de um mesmo aprendiz. Ressalta-se, assim, a importância de futuros estudos e propostas que dêem conta, através de mecanismos formais da teoria, de tal possibilidade de variação referente ao input.

\section{REFERÊNCIAS BIBLIOGRÁFICAS}

ALVES, U. K. O papel da instrução explícita na aquisição fonológica do inglês como L2: evidências fornecidas pela Teoria da Otimidade. Pelotas: Dissertação de Mestrado, UCPel, 2004.

ARCHANGELI, D. Optimality Theory: an introduction to linguistics in the 1990s. In: ARCHANGELI, D. \& LANGENDOEN. Optimality Theory: an overview. Oxford: Blackwell, 1997.

BISOL, L. A sílaba e seus constituintes. In: NEVES, Maria Helena de Moura (org.). Gramática do Português Falado - Volume VII: Novos estudos. Campinas, Editora da Unicamp, 1999, p. 701-742. BONILHA, G. F. G. Teoria da Otimidade. In: MATZENAUER, C. L. B. \& BONILHA, G. F. G. Aquisição da fonologia e teoria da otimidade. Pelotas: EDUCAT, 2003, p. 13-24.

\& MATZENAUER, C. L. B. Teoria da Otimidade e construção de hierarquias. In: MATZENAUER, C. L. B. \& BONILHA, G. F. G. Aquisição da fonologia e teoria da otimidade. Pelotas: EDUCAT, 2003, p. 167-182.

BOROWSKY, T. Word-faithfulness and the direction of assimilations. The Linguistic Review 17, 2000, p. 1-28.

CLEMENTS, G. N. The role of the sonority cycle in core syllabification. IN: KIGSTON, J. \& BECKMAN, M. (Orgs.) Papers in laboratory phonology 1. Cambridge: CUP, 1990, p. 283-333.

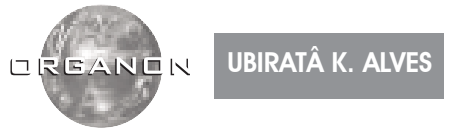

COLLISCHONN, G. A epêntese vocálica no português do sul do Brasil: análise variacionista e tratamento pela Teoria da Otimidade. Letras de Hoje, Porto Alegre, v. 35, n. 1, 2000, p. 285-318.

ELLIS, R. The structural syllabus and second language acquisition. TESOL Quarterly, v. 24, n. 1, 1993, p. 91-113.

A theory of instructed second language acquisition. In: ELLIS, N. (Ed.). Implicit and Explicit Learning of Languages. San Diego, CA: Academic Press, 1994, p. 79-114.

. The study of second language acquisition.

Oxford University Press, 1994 (b).

. Teaching and Research: Options in Grammar Teaching. TESOL Quarterly, v. 32, n. 1, 1998, p. 39-60.

FERNANDES, P. A Epêntese Vocálica na Interfonologia Português-Inglês. Pelotas: Dissertação de Mestrado. - UCPel, 1997.

A epêntese nas formas oral e escrita na Interfonologia Português/Inglês. In: MATZENAUER, C. L. Aquisição de Lingua Materna e de Língua Estrangeira - Aspectos fonéticos-fonológicos. EDUCAT, 2001, p. 235-260. GUSSENHOVEN, C. \& JACOBS, H. Understanding Phonology. London: Arnold, 1998. HAMMOND, M. The Phonology of English: A prosodic-optimality theoretic approach. Oxford University Press, 1999.

LEE, S-H. Teoria da Otimalidade e Silabificação do PB. In: IBLER, V. B., MENDES, E. A. M., OLIVEIRA, P. (eds.). Revisitações: Edição Comemorativa dos 30 anos da FALE/UFMG, Belo Horizonte, 1999, p. 143-156.

LOMBARDI, L. Why Place and Voice are different: Constraint-specific alternations in Optimality Theory. In: LOMBARDI, L. (ed.). Segmental Phonology in Optimality Theory: Constraints and Representations, 2001 (obtido através do Rutgers Optimality Archive, em 20.12.2003).

LOPEZ, B. S. The Sound Pattern of Brazilian Portuguese (Cariocan Dialect). Microfilms International, England, 1979.

McCARTHY, J. A ThematicGuide to Optimality Theory. Cambridge University Press, 2002.

MATZENAUER, C. L. B. Aquisição da linguagem e Otimidade: uma abordagem com base na sílaba. In: MATZENAUER, C. L. B \& BONILHA, G. F. G. Aquisição da fonologia e teoria da otimidade. Pelotas: EDUCAT, 2003, p. 55-66. 
, NOBRE-OLIVEIRA, D., DE MARCO, M. O comportamento da coda na aquisição do inglês como LE: um estudo com base na Teoria da Otimidade. In: III Seminário Internacional de Fonologia, Porto Alegre, 2002.

PRINCE, A. \& SMOLENSKY, P. Optimality Theory: Constraint Interaction in Generative Grammar. Technical Report 2, 1993. ROA Version 8/2002 (http://roa.rutgers.edu). Download realizado em 7 de junho de 2003.

TESAR, B. \& SMOLENSKY, P. Learnability in Optimality Theory (long version). ROA - 156, 1996 (download em 20.12.2003). . Learnability in Optimality Theory. Cambridge, MA: MIT Press, 2000.

\section{Notas:}

${ }^{2}$ Considera-se, como representante de todos os verbos do grupo A, a forma verbal lived [livd], e como representante de todos os verbos do grupo $\mathrm{B}$, a forma verbal missed [mist].

${ }^{3}$ Para a verificação do tratamento da estrutura silábica e da sonoridade em um mesmo sistema hierárquico, sugere-se a leitura de Alves (2004).

${ }^{4}$ Salienta-se que, diferentemente da hierarquia proposta por Lee (1999) para o português, em que Max não se encontra em relação de dominância referentemente a CodaCond, o presente trabalho adota, como caracterizadora da hierarquia da estrutura silábica do português, a relação Max $>>$ CodaCond, conforme Collischonn (2000).

${ }^{5}$ Para a análise a ser realizada neste capítulo, somente Dep ${ }^{\mathrm{Nuc}}$ se mostrará relevante, de modo que Dep ${ }^{\text {onset }}$ não será retratado nos tableaux que seguem. Também nos tableaux vindouros, a restrição onset será sempre respeitada por todos os candidatos a output considerados neste trabalho, não havendo a necessidade, portanto, de apresentá-la em tais tableaux.

${ }^{6}$ No tableau em questão, são apresentadas as restrições referentes à hierarquia silábica do português brasileiro que se mostraram pertinentes para a presente análise. A restrição Son, conforme será discutido posteriormente no artigo, se mostrará irrelevante para a análise aqui desenvolvida, ainda que tal restrição se oponha a seqüências consonantais não permitidas pelo PB. Tal restrição, dessa forma, não figurará nos tableaux a serem apresentados neste trabalho, o que será justificado posteriormente.

${ }^{7} \mathrm{O}$ presente trabalho considera os casos de epêntese aqui evidenciados como produtos de estratégias de reparo silábico decorrentes de uma hierarquia contendo restrições referentes exclusivamente à estrutura silábica, de modo que as formas diferentes da tida como alvo não sejam vistas como produto da interação de restrições pertencentes à família Align.

${ }^{8}$ Nos tableaux apresentados nesta seção, referentes à análise da aquisição da estrutura silábica, a questão da sonoridade da plosiva coronal final não será por enquanto considerada.

${ }^{9}$ Reconhece-se que o candidato [li $]$, com duplo apagamento e sem coda, mostrar-se-ia como ótimo se considerado entre os candidatos possíveis. A possibilidade de tal forma figurar no rol de candidatos, entretanto, foi descartada, pelo fato de não se ter verificado nenhuma manifestação da forma [lı] na produção de tais aprendizes. Uma possibilidade de explicação para o fenômeno seria estabelecer uma relação entre os apagamentos produzidos pelos aprendizes e a exigência, no inglês, de que toda a sílaba deve ser bimoraica (conforme Hammond 1999), possibilidade essa cuja verificação foge do escopo deste trabalho. A questão da saliência perceptual também não pode ser esquecida, uma vez que há uma diferença considerável entre as formas exibindo uma coda.complexa de outras formas encerradas no núcleo, que excluiria a produção de [li] ao invés de [livd]. Não é objetivo deste estudo discutir detalhadamente as diversas possibilidades de explicação para a questão. Entretanto, vistas tais hipóteses que justificariam o não-apagamento de dois segmentos, o presente trabalho se reservará o direito de excluir, do seu rol de candidatos ao posto de ótimo, formas como [li $]$.

${ }^{10} \mathrm{~A}$ restrição em questão determina que os segmentos complexos do português brasileiro devem respeitar pelo menos duas escalas de sonoridade de acordo com o Princípio da Dispersão de Sonoridade (cf. Clements 1990).

${ }^{11}$ Não serão discutidos, em função da necessidade de delimitação deste trabalho, os possíveis motivos que justificam a coexistência de formas subjacentes, para o grupo $\mathrm{B}$, anteriormente mesmo ao trabalho de intervenção pedagógica, diferentemente do que pôde ser visto para os verbos do grupo A.

${ }^{12}$ Chama-se a atenção para o fato de que as restrições mais baixas da hierarquia, que não apresentaram alteração, em termos de ranqueamento, com relação a $\mathrm{H}_{0}$, não foram aqui retratadas.

${ }^{13}$ Optou-se por representar o estrato em sua hierarquia em que Max se encontra dominando CodaCond pelo fato de ser essa a sub-hierarquia predominante, uma vez que os índices numéricos referentes à produção de tais outputs advindos da sub-hierarquia em questão se mostram como bastante superiores.

${ }^{14}$ Ressalta-se que, neste quadro, a questão da sonoridade do segmento plosivo coronal final não está sendo ainda abordada, de modo que os outputs ótimos sejam representados, convencionalmente, com o valor de sonoridade fiel ao input /d/ (como em lived [livd]), com exceção da seqüência consonantal final missed [mist], cujo segmento final está sendo representado com o traço [-son].

${ }^{15}$ Utiliza-se o símbolo --------- para representar possibilidades de input e outputs lógicos que não foram efetivamente encontradas nas manifestações dos aprendizes

${ }^{16}$ Reconhece-se que a análise de Lombardi (2001) se opõe à existência de uma restrição de marcação posicional como ] $\sigma /$ *Voice, propondo uma tipologia fatorial para a questão de vozeamento através das restrições IDOnsLar, *Lar e MaxLar. O presente trabalho, entretanto, adotará a restrição de marcação posicional pelo fato de uma análise baseada apenas nas três restrições utilizadas no trabalho de Lombardi não conseguir retratar a impossibilidade de outputs como [mizd]. Para dar conta de tal impossibilidade, seria necessário invocar outras restrições, o que tornaria a análise menos econômica.

${ }^{17}$ Para a exemplificação através de tableaux, serão aqui tomados, como exemplo representante do grupo 1, a forma lived, e como representante do grupo 2, a forma missed.

${ }^{18}$ Cabe lembrar que, no caso de dessonorizações em verbos pertencentes ao grupo 1 , ambos os segmentos obstruintes que compunham a coda complexa eram dessonorizados, conforme pode ser visto no Quadro 01.

${ }^{19}$ Não se pode esquecer, entretanto, que o português, ao contrário do alemão, permite obstruintes sonoras em coda, desde que estejam seguidas por um outro segmento também sonoro. Nesse sentido, reconhece-se, no caso do português, a atuação de uma 
outra restrição que garanta os efeitos da assimilação regressiva de sonoridade, garantindo que a sonoridade da obstruinte em coda concorde com a do onset seguinte, restrição essa que deve estar dominando a relação $] \sigma / *$ Voice $>$ Ident(Voice). Não é o objetivo do presente trabalho tratar dessa restrição, mas, sim, evidenciar que, em casos em que a fricativa de coda do português não é seguida por nenhum outro elemento, antecedendo dessa forma a pausa, a relação de dominância ] $\sigma / *$ Voice $>>$ Ident(Voice) se faz perceptível. Tal tendência se mostra bastante clara na produção de aprendizes brasileiros em estágio inicial de aquisição da língua inglesa, que costumam, também, dessonorizar todas as plosivas finais sonoras quando essas antecedem a pausa. 